\title{
A Sokhotski-Plemelj problem related to a renewable T-cell
}

\author{
EJ Vanderperre* \\ Received: 20 May 2005; Revised: 8 August 2005; Accepted: 30 August 2005
}

\begin{abstract}
We analyse the long-run availability of a technical device subjected to inspections and repair. All statistical distributions involved are general. In order to describe the random behaviour of the system, we employ a stochastic process endowed with stationary measures satisfying coupled Hokstad-type differential equations. The solution procedure is based on the theory of sectionally holomorphic functions. As an example, we consider the particular case of Coxian inspections.
\end{abstract}

Key words: Statistical reliability, stochastic process, stationary measures.

\section{Introduction}

Statistical Reliability Engineering is a topic of particular interest in Operations Research (see, for example, Shaked et al. (1990)). In this paper we introduce a (new) methodology to analyse the stochastic behaviour of a renewable $\mathbf{T}$-cell (a technical device that requires a man-machine interaction) subjected to general statistical distributions with respect to failure, inspection and repair. Our method is also applicable to modified systems.

Innovations in the field of microelectronics and micromechanics have enhanced the involvement of "smart" electro-mechanical devices in all kind of engineering applications as described by Brandin (1996). Unfortunately, no device is completely reliable. The usual "bugbears" are software failures (Gaskill \& Went (1996)), common-cause failures (Dhillon \& Yang (1997) and wear-out (Birolini (1994)). Often, a technical device requires a man-machine interaction to reveal (detect) "hidden" failures (Gnedenko et al. (1994)). For instance, a fault-tolerant electro-optical device requires regular inspections to ascertain the performance level of the unit. Therefore, human inspections are indispensable in attempts at increasing the reliability, availability, quality and safety of operational plants.

${ }^{*}$ Department of Decision Sciences, University of South Africa, P.O. Box 392, Pretoria 0003, South Africa, email: evanderperre@yahoo.com 
The reliability of complex engineering systems operating in a Markov environment, subjected to random inspections, perfect repair (Gertsbakh (1989)) or instantaneous replacements, has been analysed by several authors (see, for example, Dieulle (2002) and Mazumdar (1970)). In this paper we consider a T-cell, subjected to random inspections and perfect repair.

Note that our $\mathbf{T}$-cell is less general with regard to the structure of the e-system presented by Dieulle (2002) and Mazumdar (1970). On the other hand, the statistical structure of our $\mathbf{T}$-cell is less restrictive, since we allow general failure, inspection and repair time distributions.

In order to analyse the long-run availability of the $\mathbf{T}$-cell, we introduce a stochastic process endowed with stationary measures satisfying general Hokstad-type differential equations. The solution procedure is based on the theory of sectionally holomorphic functions. Finally, as an example, we consider the particular case of Coxian inspections.

\section{Formulation}

Consider the T-cell operating in a random environment. The $\mathbf{T}$-cell satisfies the usual conditions, i.e. independent, identically distributed random variables with finite mean and variance. The failure-free time of the $\mathbf{T}$-cell, denoted by $f$, is assumed to have a general cumulative distribution function $F(\cdot), F(0)=0$.

Suppose inspections are performed at time instants $t_{n}, n=1,2 \ldots$ where $t_{0}=0$. The interinspection times, called cycles, are supposed to be independent, identically distributed random variables with distribution $H(\cdot), H(0)=0$. The inspection cycle is denoted by $h$. Note that the inspection process is assumed to end after detection of the failed $\mathbf{T}$-cell and restarts after the instant of a repair completion. The repair time of the $\mathbf{T}$-cell, denoted by $r$, has a general cumulative distribution function $R(\cdot), R(0)=0$. Finally, we assume that $f, h, r$ are statistically independent. Characteristic functions may be formulated in terms of a complex transform variable. For instance,

$$
\mathbf{E} e^{i \omega r}=\int_{0}^{\infty} e^{i \omega x} \mathrm{~d} R(x), \quad \operatorname{Im} \omega \geq 0 .
$$

Observe that

$$
\mathbf{E} e^{-i \omega r}=\int_{-\infty}^{0} e^{i \omega x} \mathrm{~d}\{1-R((-x)-)\}, \quad \operatorname{Im} \omega \leq 0
$$

The corresponding Fourier-Stieltjes-transforms are called dual transforms. Without loss of generality with regard to $F$ and $R$, we may assume that $F, R$ are Lebesgue-absolutely continuous with bounded density functions (in the Radon-Nikodym sense) defined on $[0, \infty)$. In addition, we assume that

(i) $H^{\prime}$ is of bounded variation on $[0, \infty)$, and

(ii) $H^{\prime \prime}$ exists on $(0, \infty)$. 
Condition (i) implies that $\mathbf{E} e^{i \tau h}, \tau \in \mathbb{R}$, is Lipschitz continuous (L-continuous) at infinity, i.e.

$$
\left|\mathbf{E} e^{i \tau h}\right|=O\left(\frac{1}{|\tau|}\right),|\tau| \rightarrow \infty
$$

See Keilson (1965, page 57).

In order to describe the random behaviour of the $\mathbf{T}$-cell, we employ a stochastic process $\left\{N_{t}, t \geq 0\right\}$ with discrete state space $\{A, B, C\} \subset[0, \infty)$, characterized by the following events:

$\left\{N_{t}=A\right\}:$ The $\mathbf{T}$-cell is operative at time $t$.

$\left\{N_{t}=B\right\}:$ The $\mathbf{T}$-cell is down at time $t$.

$\left\{N_{t}=C\right\}$ : The $\mathbf{T}$-cell is under repair at time $t$.

Note that state $B$ corresponds to an unrevealed failure of the T-cell. A (vector) Markov characterization of the process $\left\{N_{t}\right\}$ is piecewise and conditionally defined by:

$\left\{\left(N_{t}, X_{t}, Y_{t}\right)\right\} \quad$ if $N_{t}=A$, i.e. if the event $\left\{N_{t}=A\right\}$ occurs, where $X_{t}$ denotes the remaining failure-free time of the $\mathbf{T}$-cell being operative at time $t$ and where $Y_{t}$ denotes the remaining inspection cycle being in progress at time $t$.

$\left\{\left(N_{t}, Y_{t}\right)\right\} \quad$ if $N_{t}=B$.

$\left\{\left(N_{t}, Z_{t}\right)\right\} \quad$ if $N_{t}=C$, where $Z_{t}$ denotes the remaining repair time of the $\mathbf{T}$-cell being under progressive repair at time $t$.

The state space of the underlying Markov process is given by

$$
\{(A, x, y), x \geq 0, y \geq 0\} \bigcup\{(B, y), y \geq 0\} \bigcup\{(C, z), z \geq 0\} .
$$

Next, we consider the $\mathbf{T}$-cell in stationary state (the so-called ergodic state) with invariant measure

$$
\left\{P_{K} ; K=A, B, C\right\} ; \Sigma_{K} P_{K}=1,
$$

where

$$
P_{K}:=\mathbf{P}\{N=K\}:=\lim _{t \rightarrow \infty} \mathbf{P}\left\{N_{t}=K \mid N_{0}=A\right\} .
$$

Finally, we introduce the measures

$$
\begin{aligned}
P_{A}(x, y) \mathrm{d} x \mathrm{~d} y & :=\mathbf{P}\{N=A, X \in d x, Y \in d y\} \\
& :=\lim _{t \rightarrow \infty} \mathbf{P}\left\{N_{t}=A, X_{t} \in d x, Y_{t} \in d y \mid N_{0}=A\right\}, \\
P_{B}(y) \mathrm{d} y & :=\mathbf{P}\{N=B, Y \in \mathrm{d} y\} \\
& :=\lim _{t \rightarrow \infty} \mathbf{P}\left\{N_{t}=B, Y_{t} \in \mathrm{d} y \mid N_{0}=A\right\}, \text { and } \\
P_{C}(z) \mathrm{d} z & :=\mathbf{P}\{N=C, Z \in \mathrm{d} z\} \\
& :=\lim _{t \rightarrow \infty} \mathbf{P}\left\{N_{t}=C, Z_{t} \in \mathrm{d} z \mid N_{0}=A\right\} .
\end{aligned}
$$

Note that, for instance, that

$$
P_{A}=\int_{0}^{\infty} \int_{0}^{\infty} P_{A}(x, y) \mathrm{d} x \mathrm{~d} y=\int_{0}^{\infty} \int_{0}^{\infty} P_{A}(x, y) \mathrm{d} y \mathrm{~d} x .
$$




\section{$2.1 \quad$ Notation}

The long-run availability of the $\mathbf{T}$-cell, denoted by $\mathcal{A}$, is defined by

$$
\mathcal{A}:=\lim _{T \rightarrow \infty} \frac{1}{T} \int_{0}^{T} \mathbf{P}\left\{N_{t}=A \mid N_{0}=A\right\} \mathrm{d} t .
$$

Hence, $\mathcal{A} \equiv P_{A}$. The indicator (function) of an event $\mathcal{E}$ is denoted by $1 \mathcal{E}$. The real line and the complex plane are denoted by $\mathbb{R}$ and $\mathbb{C}$ with obvious superscript notations $\mathbb{C}^{+}, \mathbb{C}^{-}$. For instance, $\mathbb{C}^{+}:=\{\omega \in \mathbb{C}: \operatorname{Im} \omega>0\}$. Finally, we introduce the notations $\lambda_{H}^{+}(\tau), \lambda_{F}^{+}(\tau), \lambda_{F}^{-}(\tau), \lambda_{R}^{+}(\tau), \tau \in \mathbb{R}$, where for instance,

$$
\lambda_{F}^{+}(\tau):= \begin{cases}\frac{\mathbf{E} e^{i \tau f}-1}{i \tau \mathbf{E} f}, & \text { if } \tau \neq 0 \\ 1, & \text { if } \tau=0\end{cases}
$$

and

$$
\lambda_{F}^{-}(\tau):= \begin{cases}\frac{1-\mathbf{E} e^{-i \tau f}}{i \tau \mathbf{E} f}, & \text { if } \tau \neq 0 \\ 1, & \text { if } \tau=0\end{cases}
$$

\section{Differential equations}

In order to determine the steady-state probability density functions (hence forth called the p-functions), we first construct a system of coupled steady-state differential equations based on Hokstad's supplementary variable technique, (see, for example, Attahiru and Srinivasa (2000)). For $x>0, y>0, z>0$, we obtain

$$
\begin{aligned}
\left(-\frac{\partial}{\partial x}-\frac{\partial}{\partial y}\right) P_{A}(x, y) & =P_{A}(x, 0) \frac{\mathrm{d} H}{\mathrm{~d} y}(y)+P_{C}(0) \frac{\mathrm{d} H}{\mathrm{~d} y}(y) \frac{\mathrm{d} F}{\mathrm{~d} x}(x) \\
-\frac{\mathrm{d}}{\mathrm{d} y} P_{B}(y) & =P_{A}(0, y) \\
-\frac{\mathrm{d}}{\mathrm{d} z} P_{C}(z) & =P_{B}(0) \frac{\mathrm{d} R}{\mathrm{~d} z}(z) .
\end{aligned}
$$

\section{Solution procedure}

It should be noted that the above equations are well adapted to an integral transformation. The integrability of the p-functions and their corresponding derivatives implies that each p-function vanishes at infinity irrespective of the asymptotic behaviour of the underlying density functions of $h, f, r$. Applying a routine Fourier-transform technique to the differential equations reveals that

$$
\begin{aligned}
& i(\omega+\eta) \mathbf{E}\left(e^{i \omega X} e^{i \eta Y} \mathbf{1}\{N=A\}\right) \\
&+\left(1-\mathbf{E} e^{i \eta h}\right) \int_{0}^{\infty} e^{i \omega x} P_{A}(x, 0) \mathrm{d} x \\
&+ i \eta \mathbf{E}\left(e^{i \eta Y} \mathbf{1}\{N=B\}\right)+P_{B}(0)=P_{C}(0) \mathbf{E} e^{i \eta h} \mathbf{E} e^{i \omega f} \\
& \text { and } \quad i \zeta \mathbf{E}\left(e^{i \zeta Z} \mathbf{1}\{N=C\}\right)+P_{C}(0)=P_{B}(0) \mathbf{E} e^{i \zeta r} .
\end{aligned}
$$


Substituting $\zeta=0$ into (2) and $\eta=0$ into (1) yields

$$
\begin{aligned}
\mathbf{E}\left(e^{i \zeta Z} \mathbf{1}\{N=C\}\right) & =P_{C}(0) \mathbf{E} r \lambda_{R}^{+}(\zeta), \quad \operatorname{Im} \zeta \geq 0 \\
\text { and } \quad \mathbf{E}\left(e^{i \omega X} \mathbf{1}\{N=A\}\right) & =P_{B}(0) \mathbf{E} f \lambda_{F}^{+}(\omega), \quad \operatorname{Im} \omega \geq 0
\end{aligned}
$$

Since $P_{B}(0)=P_{C}(0)$, we have that $P_{C}=P_{B}(0) \mathbf{E} r$ and $P_{A}=P_{B}(0) \mathbf{E} f$. Substituting $\omega=-\tau, \eta=\tau$ for some $\tau \in \mathbb{R}$ into (1) yields the Hilbert equation

$$
\psi^{+}(\tau)-\psi^{-}(\tau)=\varphi(\tau), \tau \in \mathbb{R}
$$

where

$$
\begin{aligned}
\psi^{+}(\tau) & := \begin{cases}\frac{i \tau}{\mathbf{E} e^{i \tau h}-1} \mathbf{E}\left(e^{i \tau Y} \mathbf{1}\{N=B\}\right)-P_{B}(0), & \text { if } \tau \neq 0, \\
-P_{B}(0)+P_{B} / \mathbf{E} h, & \text { if } \tau=0,\end{cases} \\
\psi^{-}(\tau) & :=\int_{0}^{\infty} e^{-i \tau x} P_{A}(x, 0) \mathrm{d} x, \\
\varphi(\tau) & :=P_{B}(0) \mathcal{K}(\tau), \text { and } \\
\mathcal{K}(\tau) & := \begin{cases}\frac{\mathbf{E} e^{-i \tau f}-1}{\mathbf{E} e^{i \tau h}-1} \mathbf{E} e^{i \tau h}, & \text { if } \tau \neq 0, \\
-\mathbf{E} f / \mathbf{E} h, & \text { if } \tau=0 .\end{cases}
\end{aligned}
$$

Note that by the Riemann-Lebesgue theorem (see, for example, Apostol (1998)),

$$
\lim _{|\tau| \rightarrow \infty} \psi^{+}(\tau)=\lim _{|\tau| \rightarrow \infty} \psi^{-}(\tau)=\lim _{|\tau| \rightarrow \infty} \varphi(\tau)=0 .
$$

Hence, $\psi^{+}, \psi^{-}$and $\varphi$ are continuous on $\mathbb{R}$ and at infinity. Equation (3) constitutes a Sokhotski-Plemelj problem on $\mathbb{R}$ solvable by means of the theory of sectionally holomorphic functions (see, for example Roos (1969)). First, note that $\mathcal{K}^{\prime}(\tau)$ is bounded on $\mathbb{R}$. Hence, by the mean value theorem (see, for example, Apostol (1998)), $\mathcal{K}(\tau)$ is $\mathbf{L}$-continuous on $\mathbb{R}$. Moreover, $\mathcal{K}(\tau)$ is L-continuous at infinity. Consequently, the Cauchy-type integral

$$
\frac{1}{2 \pi i} \int_{\Gamma} \mathcal{K}(\tau) \frac{\mathrm{d} \tau}{\tau-\omega}, \quad \omega \in \mathbb{C}
$$

exists for all $\omega \in \mathbb{C}$ (real or complex), provided that the singular integral

$$
\frac{1}{2 \pi i} \int_{\Gamma} \mathcal{K}(\tau) \frac{\mathrm{d} \tau}{\tau-u}, \quad u \in \mathbb{R}
$$

is defined as a Cauchy principal value in double sense, (see Vanderperre (2000) for a precise definition of $\Gamma$ ).

Moreover, the Cauchy-type integral represents a sectionally holomorphic function in $\mathbb{C}$, vanishing at infinity. A straightforward application of the theory of sectionally holomorphic functions entails that

$$
\psi^{+}(\omega)=\frac{1}{2 \pi i} \int_{\Gamma} \varphi(\tau) \frac{d \tau}{\tau-\omega}, \quad \omega \in \mathbb{C}^{+}
$$


or more explicitly,

$$
\mathbf{E}\left(e^{i \omega Y} \mathbf{1}\{N=B\}\right)=\mathbf{E} h P_{B}(0) \lambda_{H}^{+}(\omega)\{1+\kappa(\omega)\}, \quad \omega \in \mathbb{C}^{+},
$$

where

$$
\kappa(\omega):=\frac{1}{2 \pi i} \int_{\Gamma} \mathcal{K}(\tau) \frac{\mathrm{d} \tau}{\tau-\omega}, \quad \omega \in \mathbb{C}^{+} .
$$

Equation (4) yields $P_{B}=\mathbf{E} h P_{B}(0)(1+\kappa)$, where

$$
\kappa:=\lim _{\substack{\omega \rightarrow 0 \\ \omega \in \mathbb{C}^{+}}} \kappa(\omega) .
$$

Applying the Sokhotski-Plemelj formula, it follows that

$$
\kappa=\frac{1}{2} \mathcal{K}(0)+\frac{1}{2 \pi i} \int_{\Gamma} \mathcal{K}(\tau) \frac{\mathrm{d} \tau}{\tau} .
$$

We summarize the results of this section as

$$
\left\{\begin{array}{l}
P_{A}=P_{B}(0) \mathbf{E} f, \\
P_{B}=P_{B}(0)(1+\kappa) \mathbf{E} h, \\
P_{C}=P_{B}(0) \mathbf{E} r
\end{array}\right.
$$

Invoking the identity $\sum_{K} P_{K}=1$, reveals that $P_{B}(0)=1 /(\mathbf{E} f+\mathbf{E} r+(1+\kappa) \mathbf{E} h)$. Hence,

$$
\mathcal{A}=\frac{\mathbf{E} f}{\mathbf{E} f+\mathbf{E} r+(1+\kappa) \mathbf{E} h} .
$$

\subsection{Some remarks}

Note that $\mathcal{A}$ satisfies the so-called insensitivity property with regard to $R$, i.e. $\mathcal{A}$ is $R$ insensitive. Consequently, $\mathcal{A}$ exists for an arbitrary $R$ with finite mean. Moreover, observe that the L-continuity of a characteristic function does not depend on the canonical structure (Lebesgue-decomposition) of the corresponding distribution. For instance, the L-inequality

$$
\left|\mathbf{E} e^{-i \tau_{1} f}-\mathbf{E} e^{-i \tau_{2} f}\right| \leq \mathbf{E} f\left|\tau_{1}-\tau_{2}\right|, \quad \tau_{1}, \tau_{2} \in \mathbb{R}
$$

always holds for any $f$ with finite mean. However, $\mathbf{E} e^{-i \tau f}$ with $f$ arbitrary, has no limit if $|\tau| \rightarrow \infty$. Therefore, we transform $\mathcal{K}(\tau)$ into the equivalent form $\mathcal{K}(\tau)=\mathcal{K}_{H}^{+}(\tau) \lambda_{F}^{-}(\tau)$, where

$$
\mathcal{K}_{H}^{+}(\tau):= \begin{cases}\frac{\mathbf{E} f i \tau \mathbf{E} e^{i \tau h}}{1-\mathbf{E} e^{i \tau h},}, & \text { if } \tau \neq 0 \\ -\mathbf{E} f / \mathbf{E} h, & \text { if } \tau=0\end{cases}
$$

From the identity

$$
i \tau \mathbf{E} e^{i \tau h}+H^{\prime}(0)+\int_{0}^{\infty} e^{i \tau x} H^{\prime \prime}(x) \mathrm{d} x=0, \tau \in \mathbb{R}
$$

and the relationship

$$
\int_{0}^{\infty}\left|H^{\prime \prime}(x)\right| \mathrm{d} x=\int_{0+}^{\infty}\left|\mathrm{d} H^{\prime}(x)\right|<\infty
$$


we obtain, by the Riemann-Lebesgue theorem,

$$
\lim _{|\tau| \rightarrow \infty} \mathcal{K}_{H}^{+}(\tau)=-\mathbf{E} f H^{\prime}(0)
$$

Hence, the L-continuity of $\mathcal{K}$ on $\mathbb{R}$ and at infinity holds for any $f$ with finite mean and variance. Thus, $\mathcal{A}$ also exists for an arbitrary $f$. Consequently, our initial assumptions concerning the existence of density functions $F^{\prime}$ and $R^{\prime}$ are superfluous with respect to ensuring the existence of an invariant measure, provided that our initial conditions imposed on $H$ are preserved.

\subsection{An example}

Let $H(x)=p_{1}\left(1-e^{-\lambda_{1} x}\right)+p_{2}\left(1-e^{-\lambda_{2} x}\right)$ where $p_{1}>0, p_{2}<0, p_{1} \lambda_{1}+p_{2} \lambda_{2}=0$ and without loss of generality $0<\lambda_{1}<\lambda_{2}$. Note that $p_{1}=\lambda_{2} /\left(\lambda_{2}-\lambda_{1}\right), p_{2}=-\lambda_{1} /\left(\lambda_{2}-\lambda_{1}\right), \mathbf{E} h=$ $\left(\lambda_{1}+\lambda_{2}\right) / \lambda_{1} \lambda_{2}$. The function $(1-H)^{-1}$ is log-convex. Hence, $H$ belongs to an important family of Coxian distributions with tractable engineering applications (see Vanderperre (1999)). For instance, $H$ is suitable to model the inspection cycle. Note that

$$
\mathbf{E} e^{i \tau h}=\lambda_{1} \lambda_{2} /\left(\lambda_{1}-i \tau\right)\left(\lambda_{2}-i \tau\right) .
$$

Hence,

$$
\kappa(\omega)=\frac{\lambda_{1} \lambda_{2}}{2 \pi i} \int_{\Gamma} \frac{\mathbf{E} e^{-i \tau f}-1}{\tau(\tau-\omega)} \frac{\mathrm{d} \tau}{\tau+i\left(\lambda_{1}+\lambda_{2}\right)}, \quad \omega \in \mathbb{C}^{+} .
$$

Applying Cauchy's theorem (see, for example, Roos (1969)), entails that

$$
\kappa(\omega)=\frac{1-\mathbf{E} e^{-\left(\lambda_{1}+\lambda_{2}\right) f}}{i \mathbf{E} h\left(\omega+i\left(\lambda_{1}+\lambda_{2}\right)\right)} .
$$

Hence, by (5),

$$
1+\kappa=1-\frac{1-\mathbf{E} e^{-\left(\lambda_{1}+\lambda_{2}\right) f}}{\left(\lambda_{1}+\lambda_{2}\right) \mathbf{E} h} .
$$

Finally, we introduce the random variable

$$
\alpha_{F}:=\int_{0}^{f} e^{-\left(\lambda_{1}+\lambda_{2}\right) x} \mathrm{~d} x .
$$

Note that $\alpha_{F} \leq f, \mathbf{P}$-almost surely. From the identity

$$
\mathbf{E} \alpha_{F}=\frac{1-\mathbf{E} e^{-\left(\lambda_{1}+\lambda_{2}\right) f}}{\lambda_{1}+\lambda_{2}}
$$

we obtain

$$
\mathcal{A}=\frac{\mathbf{E} f}{\mathbf{E} f+\mathbf{E} r+\mathbf{E} h-\mathbf{E} \alpha_{F}} .
$$




\section{Conclusion}

The long-run availability of a technical device, which we called a T-cell, has been analysed by a powerful methodology based on the theory of sectionally holomorphic functions. In particular, an explicit formula for the long-run availability of the $\mathbf{T}$-cell was derived in general (i.e. assuming no specific statistical distributions with regards to the interinspection and repair times) and this result was illustrated for the special case where Coxian inspections are assumed. The analysis of the time-dependent T-cell (i.e. the point availability) is still an open problem.

\section{References}

[1] Apostol TM, 1997, Mathematical analysis, Addison Wesley P.C., New York (NY).

[2] Attahiru SA, Srinivasa Rao TSS, 2000, Supplementary variable technique in stochastic models, Probability in the Engineering and Informational Sciences, 14, pp. 203-218.

[3] Birolini A, 1994, Quality and reliability of technical systems, Springer, Berlin.

[4] BRANDIN BA, The real-time supervisory control of an experimental manufacturing cell, IEEE Transactions on Robotics and Automation, 12, pp. 1-13.

[5] Dhillon BS \& YAng N, Stochastic analysis of an active standby redundant network with two types of common-cause failures, Stochastic Analysis and Applications, 15, pp. 313-325.

[6] Dieulle L, 2002, Reliability of several component sets with inspections at random times, European Journal of Operational Research, 139, pp. 96-114.

[7] Gaskill SP \& Went SRG, 1996, Safety issues in modern applications of robots, Reliability Engineering \& System Safety, 53, pp. 301-307.

[8] Gertsbakn IB, 1989, Statistical reliability theory, Marcel Dekker, New York (NY).

[9] Gnedenko B \& Ushakov IA, 1995, Probabilistic reliability engineering, John Wiley \& Sons, New York (NY).

[10] KeILson J, 1965, Green's function methods in probability theory, Griffin \& Co., London.

[11] Mazumdar M, 1970, Reliability of two-unit redundant repairable systems when failures are revealed by inspections, SIAM Journal on Applied Mathematics, 19, pp. 637-647.

[12] Roos BW, 1969, Analytic functions and distributions in physics and engineering, John Wiley \& Sons, New York (NY). 
[13] Shaked M \& Shanthikumar IG, 1990, Reliability and maintainability, pp. 666667 in Heyman DP \& Sobel MJ (EDs.), Handbook in Operations Research and Management Science, 2, North-Holland, Amsterdam.

[14] VANDERPERRE EJ, 1999, On hazard rates modelled by mixtures of exponential distributions, Belgian Journal of Operations Research, Statistics and Computer Science, 39, pp. 31-38.

[15] VANDERPERRE EJ, 2000, Long-run availability of a two-unit standby system subjected to a priority rule, Bulletin of the Belgian Mathematical Society, 7, pp. 355-364. 
